\title{
URBAN VISION DEVELOPMENT IN ORDER TO MONITOR WHEELCHAIR USERS BASED ON THE YOLO ALGORITHM
}

\author{
Ali Ahmadi ${ }^{1 *}$, Meysam Argany ${ }^{1}$, Najmeh Neysani Samany ${ }^{1}$, Mahmood Rasooli ${ }^{2}$ \\ ${ }^{1}$ Geography Faculty, University of Tehran, Tehran, Iran - (ali.ahmadi2012, argany, nneysani)@ut.ac.ir \\ ${ }^{2}$ Department of software engineering, Shahab Danesh university, Qom, Iran - rasuli.ut@gmail.com
}

KEY WORDS: Wheelchair Detection, Urban Vision, Artificial Intelligence, YOLOv3, Disability

\begin{abstract}
:
Disability has been one of the most important problems of social communities throughout the ages. As population and urbanization have grown dramatically over recent years, this problem has more and more created the gap between people with disabilities and ordinary people in terms of access to resources, social services and social partnerships. Therefore, this study attempts to demonstrate the ratio of presence of wheelchair users in a community compared to the total population of the same community and evaluate their patterns of presence in different conditions, for example, various weather conditions. For this purpose, we used the You Look Only Once version 3 (YOLOv3) algorithm which is a multilayer deep learning object detection tool to analyze and extract wheelchair users from three different sets of images taken by a camera located in an intersection proximate to a rehabilitation center in Quebec, Canada. The results show that the proportion of wheelchair users in the sample community is $7.4 \%$, while the population with mobility disabilities in Canada is $9.6 \%$.
\end{abstract}

\section{INTRODUCTION}

Disability is a dynamic, multidimensional and universal phenomenon that has been and continues to be present in all societies. This phenomenon can occur during the life of most people in a community, temporarily or permanently (World Health Organization, 2011; Avades et al., 2016). The growth and severity of this phenomenon depends on many factors, including the increase in the world population, medical and technological advances, environmental issues and access to resources, all of which have been dramatically increased in recent years considerably (Oberlink, 2006; Peters \& Cotton, 2016). On the other hand, the advancement of technology, urbanization and access to various resources has resulted in the quality of life improvement and the ease of everyday life, which has led to an increase in life expectancy and a rise in certain diseases such as diabetes (Centers for Disease Control and Prevention, 2017; Klonoff, 2009; Centers for Disease Control and Prevention, 2017). Many of these diseases are considered to be causing or intensifying the disability, for example, HIV and diabetes (WHO, 2011). Since most of these phenomena are taking place in the city and the city also occupies a large part of the world's population and, on the other hand, urbanization is expanding more intensively (Khetani et al., 2014), therefore there is a strong need to create an appropriate and smart urban environment for citizens. Many cities do not have the proper infrastructure for people with disabilities, and this creates an imbalance in the fair access to and use of urban resources and services (Forsyth, Colver, Alvanides, Woolley, \& Lowe, 2007; WHO, 2002). The environment also has a significant impact on the incidence of disability, so that in many definitions, disability is defined in relation to the environment (Botticello, Rohrbach, \& Cobbold, 2014; Gleeson, 1999). In many studies, the role of social participation and its positive impact on the brain and physical functioning of all individuals in a community, especially the elderly and people with disabilities have been identified (Ashida, Kondo, \& Kondo, 2016; a.S. et al.,
2009; J et al., 2011; Väänänen et al., 2009). One of the most important necessities in society is the mobility of individuals. Mobility is one of the most basic and essential elements for welfare and the main factor of social participation, but urban spaces and pedestrians often do not meet these needs (Iwasawa, Nagamine, Yairi, \& Matsuo, 2015). The lack of infrastructure and urban services for people with disabilities results in the isolation of this part of society and lack of their participation in the community. Therefore, this study seeks to establish a suitable framework by using the power of new innovations in YOLOv3 algorithm for monitoring these individuals and their share of the population in the community.

\section{METHODS}

Since its emergence, the $\mathrm{YOLO}^{1}$ algorithm has been one of the fastest and most accurate algorithms for identifying objects. There are three different versions of this algorithm, each one was one of the best in its time and this is approved by different papers (Huang, Pedoeem, \& Chen, 2019; J. Redmon \& Farhadi, 2016, 2017; J. S. D. R. G. A. F. Redmon, 2016; Ren, Fang, \& Djahel, 2017; Rijthoven, Swiderska-chadaj, \& Ciompi, 2018). This study was performed using the YOLOv3 algorithm and three sets of images, while each set has obtained from a different source. Figure 1 illustrates that the YOLOv3 algorithm consists of 106 layers called Darknet-53 (J. Redmon \& Farhadi, 2017) and has been changed compared to YOLOv2, such as the use of the Softmax function instead of the Sigmoid function, as well as identifying objects in three different scales using the concept of feature pyramid networks and a complex 106-layer network (Hui, 2018). The mean average precision of the algorithm with the trained data is $81.76 \%$ which is acceptable in this study.

The images used in this research are from a simple camera located at an intersection in Quebec, Canada. The reason for choosing this intersection is the proximity to a rehabilitation

\footnotetext{
* Corresponding author
}

1 You only look once 
center for people with disabilities. The data was monitored over a period of 4 months and from 6 am to $10 \mathrm{pm}$ in a 15-minute interval, resulting in a total of 60 photos per day. The obtained photographs were analyzed using the YOLOv3 algorithm, and the two desired objects, normal people and wheelchair users,

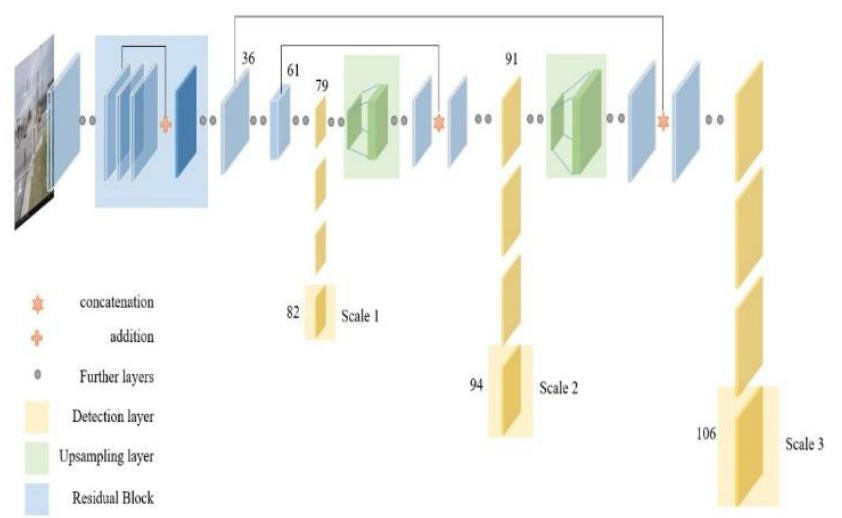

were extracted from images.

\section{RESULTS}

This study covers a period of 134 days starting on 29/03/2017 and continuing until 09/08/2017. Chart (1) shows the results obtained from these 134 days and indicates a lower percentage of wheelchair users share in the first month of the study (the first month of spring) compared to the first month of the summer, which is affected by atmospheric changes and weather condition in the area which created restrictions for wheelchair users. The results show a correlation between temperature and presence of the wheelchair users on the community. The collected data from the heat sensors placed with the camera at the intersection shows that an increase in the temperature will cause a rise in the number

Figure 1. YOLOv3 network Architecture of wheelchair users seen at the intersection. Overall, the percentage of wheelchair users participation is $7.4 \%$ compared to normal people and this ratio is only obtained from the number of wheelchair users and normal people in the photos, while the percentage of the people with mobility disabilities in Canada is $10 \%$ (Morris, Fawcett, Brisebois, \& Hughes, 2017). However, this proportion of participation of wheelchair users is also affected by the proximity of the intersection to the rehabilitation center for people with disabilities, also the accuracy of the algorithm should be taken into account.

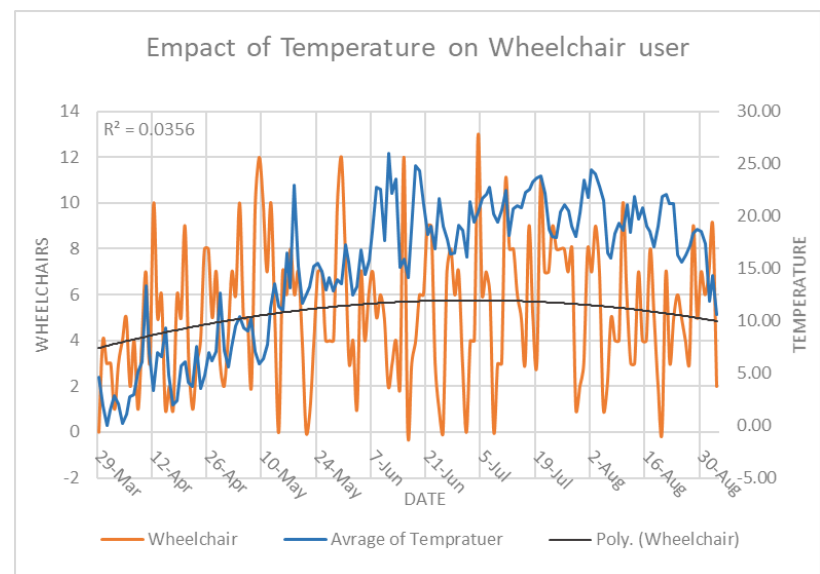

Chart 1. Relation of temperature and presence of wheelchair users
Chart (2) shows the number of wheelchair users and normal people based on different hours of the day. The correlation between these two graphs is $93.6 \%$, however, there is a considerable difference between social activities of wheelchair users and other people. As displayed in the chart, the initial peak for normal people happens around 09:00, while for wheelchair users it happens around 12:00. This difference can be caused by many reasons, for instance, the high age of wheelchair users, the lack of employment of these people or avoiding the traffic jams in the morning. The numbers in both groups start to decrease after 12:00, although by 15:00 they again start to increase. Between 16:00 and 18:00 they experience a dramatic fall, and after 18:00 they decrease steadily until 22:00.

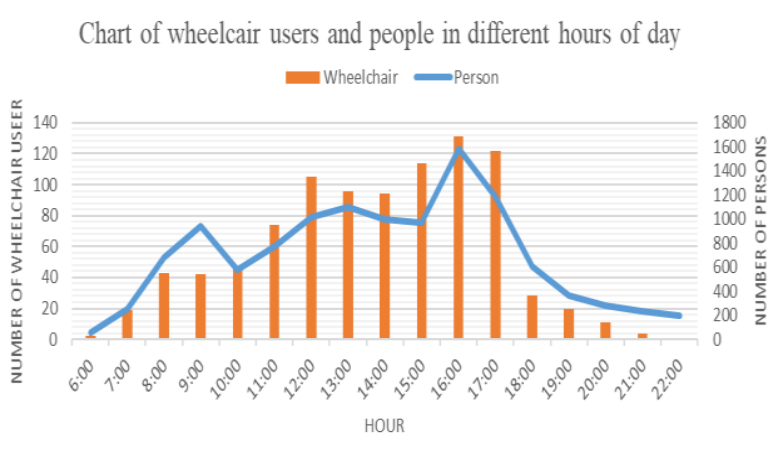

Chart 2. Number of wheelchair users and people in different hours of the day

\section{CONCLUSION}

In all societies, there are constraints for people with disabilities both in terms of structure and environmental effects. While the presence of wheelchair users can be affected by the weather condition, generally, it follows the same pattern as the presence of the normal people, i.e. when the number of normal people increases at the scene, the number of wheelchair users is also increasing almost by the same proportion. Also, the hour of the day is a determining factor in the presence of the wheelchair users which is illustrated by Chart (2). Overall, it can be said that the presence of wheelchair users and normal people follow the same pattern and the proportion of wheelchair users in the community often remains constantly around $7.4 \%$.

\section{ACKNOWLEDGMENT}

I would like to express my deep gratitude to Professor Mir Abolfazl Mostafavi, my research supervisors, for their patient guidance, enthusiastic encouragement and useful critiques of this research work.

\section{REFERENCES}

Avades, T., Doulton, F., Fajerman, M., Huovinen, K., Ito, A., Martinho, M., ... Crock, M. (2016). Global Status Report on Disability and Development | 2015, 230. Retrieved from http://www.un.org/esa/socdev/documents/disability/2016/Globa 1StatusReportonDisabilityandDevelopment.pdf

a.S., B., P.a., B., R.S., W., D.a., F., S., L., \& D.a., B. (2009). Association between late-life social activity and motor decline in older adults. Archives of Internal Medicine, 169(12), 11391146. Retrieved from 
http://www.embase.com/search/results?subaction=viewrecord\& from=export\&id=L354820022\%5Cnhttp://archinte.amaassn.org/cgi/reprint/169/12/1139\%5Cnhttp://dx.doi.org/10.1001 /archinternmed.2009.135\%5Cnhttp://sfx.hul.harvard.edu/sfx_lo cal?sid=EMBASE\&issn=0003992

Ashida, T., Kondo, N., \& Kondo, K. (2016). Social participation and the onset of functional disability by socioeconomic status and activity type: The JAGES cohort study. Preventive Medicine, 89, 121-128.

https://doi.org/10.1016/j.ypmed.2016.05.006

Botticello, A. L., Rohrbach, T., \& Cobbold, N. (2014). Disability and the built environment: An investigation of community and neighborhood land uses and participation for physically impaired adults. Annals of Epidemiology, 24(7), 545-550. https://doi.org/10.1016/j.annepidem.2014.05.003

Centers for Disease Control and Prevention, C. (2017). National Diabetes Statistics Report: Estimates of Diabetes and Its Burden in the United States. Atlanta, GA: Centers for Disease Control and Prevention; 2017. US Department of Health and Human Services, (Cdc), 2009-2012.

https://doi.org/10.1177/1527154408322560

Forsyth, R., Colver, A., Alvanides, S., Woolley, M., \& Lowe, M. (2007). Participation of young severely disabled children is influenced by their intrinsic impairments and environment. Developmental Medicine and Child Neurology, 49(5), 345-349. https://doi.org/10.1111/j.1469-8749.2007.00345.x

Gleeson, B. J. (1999). Geographies of Disability. london and New york (Vol. 18). https://doi.org/10.1016/S02642751(00)00055-X

Huang, R., Pedoeem, J., \& Chen, C. (2019). YOLO-LITE: A Real-Time Object Detection Algorithm Optimized for NonGPU Computers. Proceedings - 2018 IEEE International Conference on Big Data, Big Data 2018, 2503-2510. https://doi.org/10.1109/BigData.2018.8621865

Hui, J. (2018). Real-time Object Detection with YOLO, YOLOv2 and now YOLOv3. Medium, 1-32. Retrieved from https://medium.com/@jonathan_hui/real-time-object-detectionwith-yolo-yolov2-28b1b93e2088

Iwasawa, Y., Nagamine, K., Yairi, I. E., \& Matsuo, Y. (2015). Toward an automatic road accessibility information collecting and sharing based on human behavior sensing technologies of wheelchair users. Procedia Computer Science, 63, 74-81. https://doi.org/10.1016/j.procs.2015.08.314

J, A., K, K., H, H., SV, S., C, M., N, K., ... K, O. (2011). Assessing the association between all-cause mortality and multiple aspects of individual social capital among the older Japanese. BMC Public Health, 11, 499. Retrieved from http://queens.ezp1.qub.ac.uk/login?url=http://ovidsp.ovid.com/o vidweb.cgi?T $=\mathrm{JS} \& \mathrm{CSC}=\mathrm{Y} \& \mathrm{NEWS}=\mathrm{N} \& \mathrm{PAGE}=$ fulltext $\& \mathrm{D}=\mathrm{m}$ edl\&AN=21702996

Kathuria, A. (2019). What's new in YOLO v3 ? YOLO v3 : Better, not Faster, Stronger. Medium, 1-14.

Khetani, M., Marley, J., Baker, M., Albrecht, E., Bedell, G., Coster, W., ... Law, M. (2014). Validity of the Participation and Environment Measure for Children and Youth (PEM-CY) for
Health Impact Assessment (HIA) in sustainable development projects. Disability and Health Journal, 7(2), 226-235. https://doi.org/10.1016/j.dhjo.2013.11.003

Klonoff, D. C. (2009). The Increasing Incidence of Diabetes in the 21st Century. Journal of Diabetes Science and Technology, 3(1), 1-2. https://doi.org/10.1177/193229680900300101

Oberlink, M. (2006). Livable communities for adults with disabilities. Policy Brief (Center for Home Care Policy and Research (U.S.)), (29), 1-6. Retrieved from http://www.ncbi.nlm.nih.gov/pubmed/16983804

Peters, K., \& Cotton, A. (2016). Environmental, structural and process barriers in breast cancer screening for women with physical disability: A qualitative study. Radiography, 22(3), e184-e189. https://doi.org/10.1016/j.radi.2016.03.005

Redmon, J., \& Farhadi, A. (2016). YOLO9000: Better, Faster, Stronger. 2017 IEEE Conference on Computer Vision and Pattern Recognition (CVPR), 6517-6525.

https://doi.org/10.1109/CVPR.2017.690

Redmon, J., \& Farhadi, A. (2017). YOLOv3: An Incremental Improvement. In 2017 IEEE Conference on Computer Vision and Pattern Recognition (CVPR) (pp. 6517-6525). IEEE. https://doi.org/10.1109/CVPR.2017.690

Redmon, J. S. D. R. G. A. F. (2016). (YOLO) You Only Look Once. CVPR Proceedings, 1-10. https://doi.org/10.1109/CVPR.2016.91

Ren, P., Fang, W., \& Djahel, S. (2017). A novel YOLO-Based real-time people counting approach. In 2017 International Smart Cities Conference (ISC2) (pp. 1-2). IEEE. https://doi.org/10.1109/ISC2.2017.8090864

Rijthoven, M. Van, Swiderska-chadaj, Z., \& Ciompi, F. (2018). You Only Look on Lymphocytes Once. Medium, (Midl), 6-8.

Väänänen, A., Murray, M., Koskinen, A., Vahtera, J.,

Kouvonen, A., \& Kivimäki, M. (2009). Engagement in cultural activities and cause-specific mortality: Prospective cohort study. Preventive Medicine, 49(2-3), 142-147. https://doi.org/10.1016/j.ypmed.2009.06.026

World Health Organization. (2002). Towards a Common Language for Functioning, Disability and Health ICF. International Classification, 1149, 1-22. https://doi.org/WHO/EIP/GPE/CAS/01.3

World Health Organization. (2011). World report on child injury prevention. Injury Prevention: Journal of the International Society for Child and Adolescent Injury Prevention, 14(1), 69. https://doi.org/10.1136/ip.2007.018143

Morris, S., Fawcett, G., Brisebois, L., \& Hughes, J. (2017). A demographic, employment and income profile of Canadians with disabilities aged 15 years and over. Retrieved from https://www150.statcan.gc.ca/n1/pub/89-654-x/89-654x2018002-eng.htm 